\title{
AVALIAÇÃO MECÂNICA E ELÉTRICA DE LIGAS AI-Fe-Zr PARA FINS ELÉTRICOS APÓS ENVELHECIMENTO NATURAL DE 18 MESES.
}

\author{
Francisco Andrey Jucá Cavalcante ${ }^{1}$ \\ Ulysses Rodrigues dos Prazeres ${ }^{2}$ \\ José Tavares Machado Neto ${ }^{3}$ \\ Hélido Gleidson de Oliveira Sena ${ }^{4}$ \\ José Maria do Vale Quaresma ${ }^{5}$
}

\begin{abstract}
Resumo: Em virtude da crescente busca por ligas mais eficientes para transmissão [Tx] e distribuição [Dx] de energia elétrica, foram investigadas as características mecânicas e elétricas antes e após 18 meses de envelhecimento natural de ligas do sistema $\mathrm{Al}-\mathrm{Fe}-\mathrm{Zr}$ produzidas em laboratório em presença de argônio. Partindo-se do Al-EC ajustando-se o Fe aos níveis aconselhado pela literatura especializada, para em seguida variar-se o $\mathrm{Zr}$ em $[0,0238 ; 0,025 ; 0,0289,0,0345$ e 0,038$] \%$. As ligas assim constituídas foram solidificadas em um molde na forma de "U", bi-partido, com $22 \mathrm{~mm}$ de diâmetro interno e $300 \mathrm{~mm}$ de altura e temperatura de vazamento de $750 \mathrm{oC}$. A peça fundida foi usinada para o diâmetro de $11 \mathrm{~mm}$ e trabalhada até o de $2,89 \mathrm{~mm}$. Os fios foram finalmente submetidos a ensaios de condutividade elétrica e tração, antes e após tratamento a $230 \mathrm{oC}$ por $1 \mathrm{~h}$ (teste de termorresistividade) e, as fraturas sendo analisadas via MEV. Cada ensaio foi executado com repetibilidade de 3 CP's. Após 18 meses todos os ensaios foram repetidos. Os resultados sugerem que o aumento de $\mathrm{Zr}$ provoca aumento no LRT e queda de condutividade, após o T.T.. Entretanto, quando submetidas ao teste de Termorresistividade, os resultados variaram dentro do limite de 10\%, indicando comportamento termorresistente. Após 18 meses as ligas apresentaram discretas variações nos valores do LRT e da condutividade elétrica antes e após o teste de termorresistividade. As fraturas geradas foram analisadas via MEV. Avaliando-se dessa forma a passividade das ligas e seu potencial como liga termorresistente em virtude do teor de $\mathrm{Zr}$.
\end{abstract}

Palavras Chaves: Ligas Termorresistentes, Caracterização Mecânica, Caracterização Elétrica, Análise de Fratura.

Abstract: Due to the increasing search for more efficient alloys for transmission and distribution of electric energy, the mechanical and electric characteristics were investigated before and after 18 months of natural aging of alloys of the system Al-fe-Zr produced at laboratory in presence of argon. Breaking of the Al-EC being adjusted the Faith at the levels advised by the specialized literature, for soon afterwards to vary $\mathrm{Zr}$ in $[0,0238$; $0,025 ; 0,0289,0,0345$ and 0,038$] \%$. These alloys had been made cast on mold in the form of "U", bipartite, with $22 \mathrm{~mm}$ of internal diameter and $300 \mathrm{~mm}$ of height and temperature of emptying of 7500C. The casting was machined for the diameter of $11 \mathrm{~mm}$ and worked up to achieve the one of $2,89 \mathrm{~mm}$. The wires finally were submitted on electric resistance and tension tests, before and after heat treatment $230{ }^{\circ} \mathrm{C}$ during $1 \mathrm{~h}$ (heat-resistive test) and the specimens obtained by tension test were analyzed through MEV. Each test was executed with repeatability of 3 specimens. After 18 months all tests were repeated. The results suggest that the increase of $\mathrm{Zr}$ content provokes the increase of LRT and decrease the power conductivity, after heat treatment. However, when it submitted to the test of heatresistive the results varied in $10 \%$ limit, indicating behavior heat-resistant of alloys. After 18 months the alloys had shown discrete variations in the values of the LRT and the power conductivity before and after the heat-resistive test. The fractures obtained had been

\footnotetext{
1,2,3 UFPA Departamento de Engenharia Mecânica

4,5 Programa de Pós Graduação do Centro Tecnológico da UFPA

ENGEVISTA, v. 8, n. 2, p. 69-76, dezembro 2006
} 
analyzed by MEV. Evaluating by this way, passivity and potential as heat-resistant alloys, due to Zr content.

Key words: Heat-resistant alloys, Mechanical Characterization, Power Characterization, Analysis of Fracture.

\section{INTRODUÇÃO}

A problemática científicometalúrgico envolvida no transporte da energia elétrica nos tempos modernos, a procura constante por novas tecnologias que potencializem a eficiência e confiabilidade dos projetos de linhas de transmissão e distribuição é alvo de estudos em todo o mundo. Nesse contexto se insere o GPEMAT/DEM/UFPA que através de uma parceria com o GRUPO ALUBAR por intermédio da FADESP, desenvolve pesquisas avançadas na área dos condutores elétricos de $\mathrm{Al}$, que é de especial importância para o nosso estado, por ser um expressivo produtor da cadeia básica do $\mathrm{Al}$ em forte expansão na produção de cabos elétricos.

$\mathrm{Na}$ vanguarda de ligas para transmissão e distribuição de energia elétrica estão aquelas construídas com cabos condutores de Al-EC com alma de aço. Entretanto, uma necessidade crucial de coloca que é a de se estudar a capacidade térmica dos condutores aéreos, uma vez que a temperatura do condutor é o parâmetro fundamental na operação de uma linha aérea de transmissão. Essa temperatura depende da corrente elétrica, das características elétricas do condutor e de parâmetros climatológicos tais como a velocidade do vento e intensidade da radiação solar. O ganho de calor por efeito Joule é diretamente proporcional à corrente na qual o condutor está submetido, Miranda (1). E, desta forma podem ser buscadas opções de unir ao alumínio alguns elementos que lhe possibilite formar ligas metálicas, como o $\mathrm{Zr}$, que suportem maiores temperaturas de operação sem deteriorar suas propriedades mecânicas e elétricas. Conseqüentemente um cabo montado com fios construídos com esta liga, além de apresentar maior ampacidade, pois suportando maior temperatura poderá suportar maior efeito Joule, adquire significativo aumento de confiabilidade pois a linha terá maior capacidade ao suportar variações de corrente elétrica seja por picos de demanda ou por fenômenos atmosféricos indesejados como descargas elétricas.

Um condutor termorresistente (CTT) pode transportar até $50 \%$ mais energia do que os cabos tradicionais CAA (Cabo de Al com Alma de Aço), colocando-se ideal para a construção de novas linhas ou recapacitação de linhas sobrecarregadas. Podendo até utilizar a infra-estrutura já existente, pois além de atender a segurança ambiental quanto aos riscos decorrentes dos efeitos de campos eletromagnéticos satisfaz as condições de segurança operacionais e até aumentandoas. Segundo Domingues (2) a recapacitação de uma linha, com cabos construídos com estas ligas, pode ser feita com desligamento parcial de apenas um dos circuitos, não ocasionando interrupção no fornecimento de energia.

A aplicação da liga TAL em climas tropicais é perfeitamente adaptável, uma vez que, os valores de esticamento do cabo podem ser ampliados para compensar o aumento da flecha em alta temperatura de projeto, sem perda de confiabilidade da linha. O aumento da capacidade de transmissão, proporcionada pela utilização da liga TAL, o torna viável economicamente, uma vez que, as condições e restrições de projeto são atendidas, isto é, utilizam-se as mesmas estruturas e flechas do cabo de Al-1350. O custo total do cabo de liga TAL é $10 \%$ maior, mas o aumento no carregamento em $60 \%$ já justifica sua aplicação, Nascimento (3).

A expectativa, das companhias elétricas e do Ministério das Minas e Energia, é que os cabos $100 \%$ de $\mathrm{Al}$ irão tomar espaço dos cabos com alma de aço 
assim como o $\mathrm{Al}$ substituiu o $\mathrm{Cu}$, em decorrência dos avanços no desenvolvimento de ligas cada vez mais fortes com maior capacidade de transmissão que os cabos tradicionais, além de não possuírem um constituinte de deterioração mais rápida que é o aço.

Motivado por esse contexto, este trabalho descreve um estudo com base na modificação da liga Al-EC que é basicamente $\mathrm{Al}$ puro, com diferentes teores de $\mathrm{Zr}$ e Fe constante na procura por uma liga com características termorresistentes, envolvendo análises laboratoriais vitais na caracterização das propriedades importantes aos condutores de transmissão e distribuição, tais como tração, condutividade elétrica e tratamentos térmicos.

\section{MATERIAIS E MÉTODOS}

As ligas foram obtidas por fundição direta a partir da temperatura de $780^{\circ} \mathrm{C}$ para se chegar, com a injeção de Argônio para a eliminação das impurezas e do gás Hidrogênio, a $750^{\circ} \mathrm{C}$ no momento do vazamento. As cinco ligas foram identificadas como $\mathrm{A}[0,0238] \%$; $\mathrm{B}[0,0251] \%$; $\mathrm{C}[0,0289] \%$; $\mathrm{D}[0,0345]$ e $\mathrm{E}[0,038] \%$ de $\mathrm{Zr}$. O teor de Fe foi mantido como aconselhado pela literatura especializada em todas as ligas. As composições foram confirmadas em um espectrômetro óptico SPECTRO de propriedade da ALUBAR de uso contínuo no monitoramento do processo industrial.

A Figura 01 apresenta uma seqüência na qual estão ilustrados os projetos do molde, o ato do Vazamento e do desmoldar para a retirada do perfil obtido.

Os perfis "U" obtidos como mostrado na Figura 1, depois de desmoldados passam por uma seqüência de operações de mudança de forma até o fio com o diâmetro final desejado, como mostra a Figura 2.

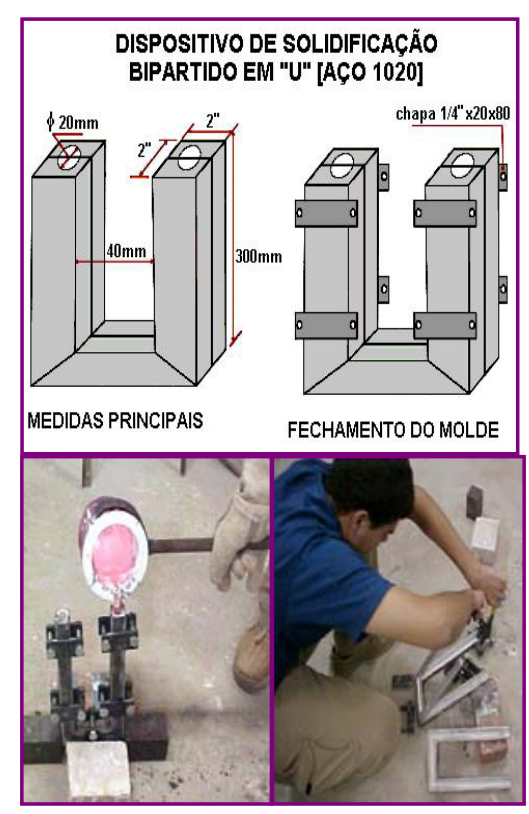

Figura 1 - Seqüência ilustrativa das operações para a obtenção do perfil “U”.

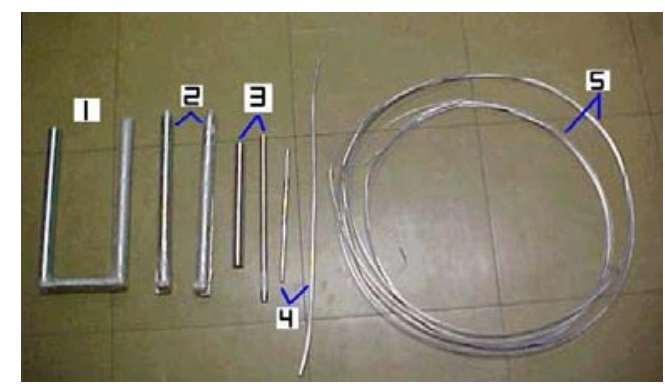

Figura 2 - Seqüência alto explicativa das etapas operacionais até a obtenção do diâmetro de 2,89mm desejado [Norma Linnet].

1- Perfil "U" com 300mm de comprimento e $20 \mathrm{~mm}$ de diâmetro; 2- Perfil "U" desmembrado em duas "pernas"; 3- As "Pernas" usinadas de $20 \mathrm{~mm}$ para $11 \mathrm{~mm}$ de diâmetro; 4- CP's laminados a frio de $11 \mathrm{~mm}$ para $4 \mathrm{~mm}$ de diâmetro em um laminador elétrico MENAC de seção circular; 5- CP's trefilados à frio até o diâmetro de 2,89mm atendendo a norma de cabos LINNET gerando os fios que serão utilizados em todos os ensaios.

\section{RESULTADOS E DISCUSSÕES}

Os condutores de alumínio convencionais [Al-1350 ou Al-EC] podem operar em um regime contínuo de trabalho a uma temperatura máxima de $75^{\circ} \mathrm{C}$, pois acima desta inicia-se o processo de 
recozimento e a conseqüente deterioração de suas características mecânicas e elétricas. As ligas de Alumínio com o Zircônio se diferenciam destas pelas suas características de recozimento e fluência em altas temperaturas que são significativamente melhores. Com o desenvolvimento destas ligas, ampliouse este limite mediante temperaturas de trabalho de até $150^{\circ} \mathrm{C}$, sem deterioração de características como tração e condutividade elétrica.

Experimentos comparativos entre uma liga termorresistente [TAL] e o Al-1350, quanto à sensibilidade ao recozimento, realizados pela Furukaua (4), estão dispostos a seguir e confirmam a maior capacidade da liga TAL em manter sua integridade em temperaturas elevadas.
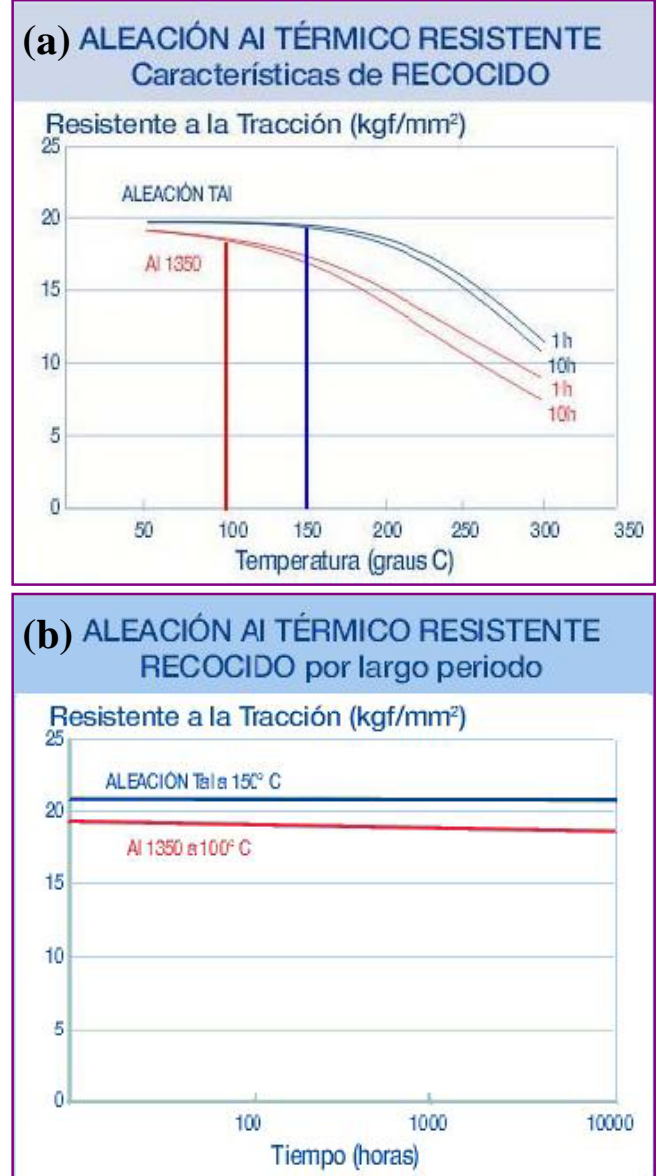

Figura 3: Resposta do LTR com a temperatura para dois tempos de tratamento (a) e LRT em função do tempo de tratamento (b).
Note que na figura 3a, torna-se visível que a perda de LRT na liga TAL é mínima até $150^{\circ} \mathrm{C}$ em quanto que na liga 1350 isso se inicia antes de $100^{\circ} \mathrm{C}$. Este gráfico justifica o teste de $230^{\circ} \mathrm{C}$ por uma hora, proposto pela COPEL já que ele deflagra perda de LRT gerada pelo recozimento no material, podendo assim confirmar ou não um comportamento termorresistente.

A Figura 4 apresenta os resultados dos ensaios de tração para a liga TAL-R3 produzida a poucos meses pelo GPEMAT obedecendo a mesma seqüência de fabricação das ligas atualmente em estudo, diferindo apenas quanto ao diâmetro $(4,77 \mathrm{~mm})$. Nesta liga foram efetuados ensaios semelhantes aos efetuados pela FURUKAWA, e a referida figura faz uma comparação entre os resultados obtidos nos dois experimentos.

Analisando-se a Figura 4 nota-se que até a temperatura de $150^{\circ} \mathrm{C}$, após 1 e 10 horas de tratamento, a tração permanece quase que inalterada (Figura 4a), começando a cair após $150^{\circ} \mathrm{C}$, o que está de acordo com o gráfico da Figura 4b, divulgado pela FURUKAWA, indicando que a faixa de $\mathrm{Zr}$ das ligas em estudo devem resultar em um material com propriedades promissoras quanto à termorresistividade.

Os valores de LRT encontrados nas ligas produzidas, para este estudo, estiveram próximos do Al-1350 e mostram nítido aumento do LRT mediante o aumento do teor de $\mathrm{Zr}$, tanto nas amostras sem o tratamento como nas tratadas termicamente e após o envelhecimento natural de 1,5 ano.

A perda de LRT ocasionada pelo tratamento térmico se dá em virtude do sensível alívio de tensões provenientes do processo fabricação dos CP's, que gera grande nível de encruamento com a laminação e trefilação. Esta queda é um fenômeno esperado e que esteve dentro da expectativa para uma liga termorresistente, pois este foi na ordem de $8 \%$. Este tratamento foi idealizado com base em um teste exigido pela COPEL (Companhia Paranaense de Energia), no qual as ligas de alumínio termorresistentes não devem apresentar variação maior que $10 \%$ nos valores dos ensaios de tração quando 
submetidos a temperatura de $230^{\circ} \mathrm{C}$ por 1hora, funcionando como um teste de termorresistividade utilizado neste trabalho.

Esta exigência esta de acordo com o proposto por Robson (5) e Morere (6) em que a baixa solubilidade do $\mathrm{Zr}$ no Al possibilita a precipitação de partículas finas de $\mathrm{Al}_{3} \mathrm{Zr}$, antes do início da homogeneização, resistentes à decomposição e ao crescimento de grão que podem controlar a estrutura dos grãos e sub-grãos durantes a operação, tanto na laminação à quente como na dissolução no tratamento térmico, elevando assim a temperatura de início desses processos, conferindo dessa forma a característica de termorresitividade a estas ligas pela maior estabilidade estrutural. Assim como a microsegregação, a composição química da liga também pode contribuir para o controle da distribuição final das partículas. Por outro lado, Ranganathan (7) comenta que evidências experimentais sugerem que a composição da liga pode ter um efeito significativo na cinética de precipitação do $\mathrm{Al}_{3} \mathrm{Zr}$ em ligas em ligas multicomponentes, comparado ao verificado no binário $\mathrm{Al}-\mathrm{Zr}$ e apresentando como exemplo, cobre, magnésio e zinco aceleram o processo de precipitação da fase $\mathrm{Al}_{3} \mathrm{Zr}$. Por isso é muito útil prever as mudanças na composição da liga sobre a precipitação dos dispersóides.

A primeira providência a ser tomada é a verificação ao atendimento de sugestões da COPEL de que a liga para ser considerada termorresistente deve apresentar valores do LRT, antes e após permanência a $230^{\circ} \mathrm{C}$ por 1 hora, que difiram entre si em até $10 \%$. A diferença superior a $10 \%$ observada para os valores do LRT para a liga " $D$ " $(0,0345 \% \mathrm{Zr})$ podem ser atribuídos a algum erro de execução dos experimentos. Entende-se, entretanto, que estes valores não inviabilizam a evidência de que o LRT cresce com o crescimento do teor de $\mathrm{Zr}$ nestas ligas.
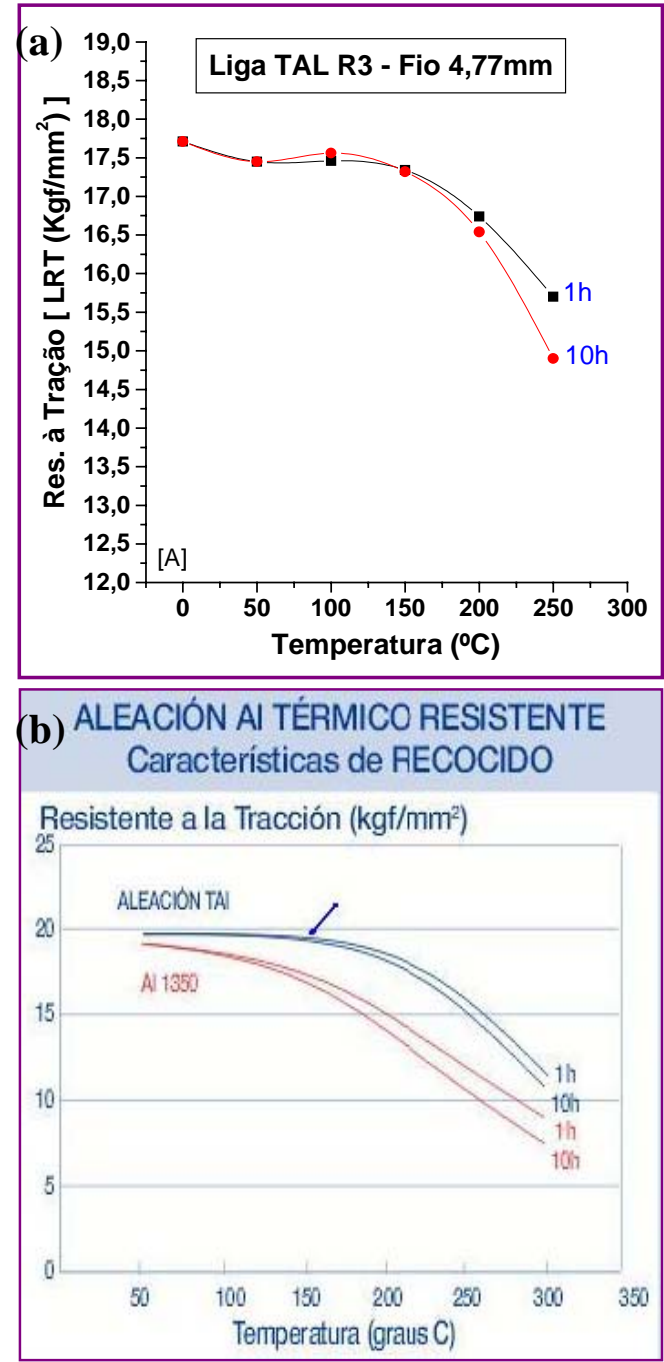

Figura 4: Curvas de Resistência à Tração para a liga TAL. (a) Gráfico experimental de resistência à tração da liga TAL R3, obtido pelo GPEMAT. (b) Gráfico do recozimento da Furukawa, a seta indica o início em que LRT começa a cair.

As Figuras 5 e 6, a seguir, mostram relacionadamente a micrografia $(1500 x)$ e o valor de LRT de cada liga produzida antes e após o teste de termorresistividade sem envelhecimento natural e com envelhecimento natural de 1,5 ano respectivamente. 


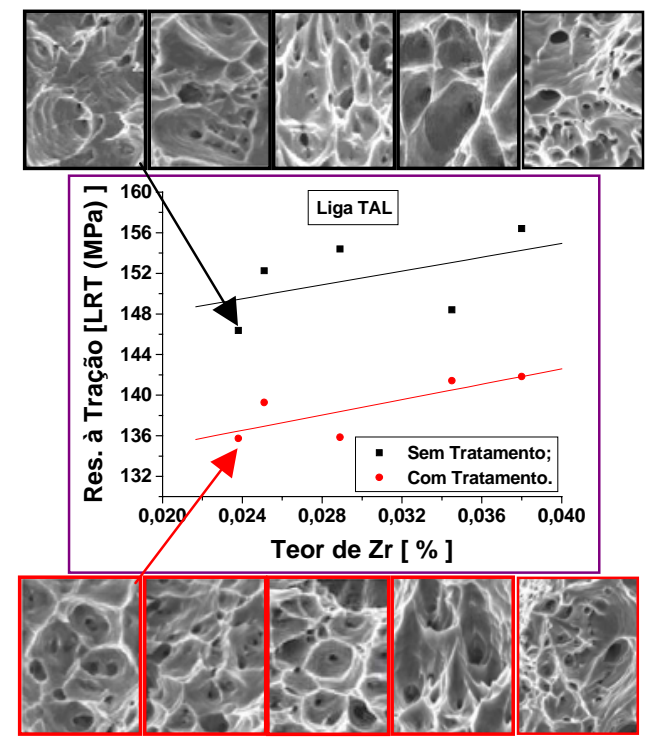

Figura 5 - Comparação entre as micrografias das ligas e os LRT antes e após o teste de termorresistividade, sem envelhecimento natural.

Os valores plotados, na Figura 5, mostram uma tendência de crescimento de LRT mediante o aumento do teor de $\mathrm{Zr}$ nas ligas, tanto para as ligas submetidas ao teste de termorresistividade como para aquelas que não foram submetidas ao teste. Este desempenho era esperado conforme estudos preliminares já executados pelo GPEMAT em teores diferentes.

Entretanto, a presença das fotomicrografias permitem emitir os seguintes comentários. Ao se comparar às espessuras das paredes das microcavidades antes e depois da permanência a $230^{\circ} \mathrm{C}$ por 1 hora, verifica-se que a espessura dos dimples nas ligas pouco mudam. Aparentemente não modificam significativamente e permanecem muito delgadas, podendo estar indicando baixa mobilidade/precipitação que estão de acordo com (5) e (6). Não obstante, nos dois casos as microcavidades presentes apresentam-se profundas e bastante abertas. A avaliação em relação à elevação do teor de $\mathrm{Zr}$ das ligas permite verificar que a liga com $0,038 \% \mathrm{Zr}$ apresenta as microcavidades com paredes mais espessas, dimples mais espessos. As ligas envelhecidas não diferiram significativamente das ligas não envelhecidas quanto ao aspecto da micrografia, apresentando dimples semelhantes, sugerindo estabilidade ao longo do tempo.

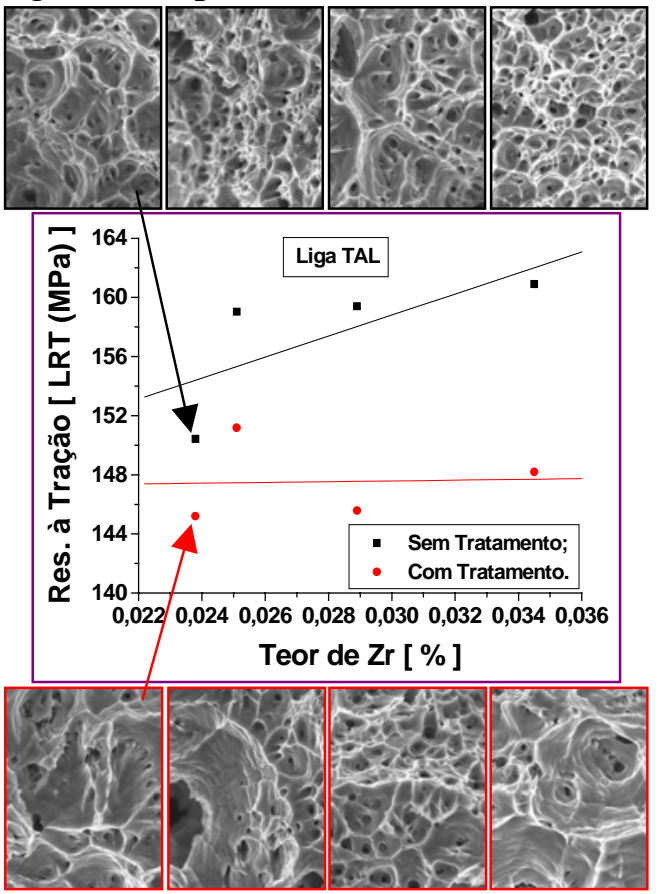

Figura 6 - Comparação entre a micrografia das ligas e os LRT antes e após o teste de termorresistividade, com envelhecimento natural de 1,5 ano.

A avaliação da Figura 6 permite inferir que o envelhecimento natural de 1,5 ano trouxe discreto aumento de LRT para as ligas, sugerindo a provável formação de um precipitado que venha a endurecer o material. As ligas envelhecidas, ao serem submetidas ao teste de termorresistividade, apresentaram menor perda de LRT que as não envelhecidas, ficando em torno de $6 \%$. Mesmo assim, a análise das microcavidades aponta para uma variação estrutural do sentido do cresciento de grão.

Nos ensaios de condutividade elétrica, cujos valores encontram-se plotados na Figura 7, notou-se uma discreta diminuição da condutividade mediante o aumento de teor de $\mathrm{Zr}$, tanto nas ligas tratadas como não tratadas termicamente. Os valores de condutividade medidos nas ligas se mantiveram bastante próximos (em torno de 60,2 IACS). Sabendo que os cabos de Al-1350 com alma de aço devem ter condutividade de pelo menos 60,6 IACS, NBR 7272/1988 (8), as ligas produzidas neste trabalho apresentaram-se bastante próximas. 

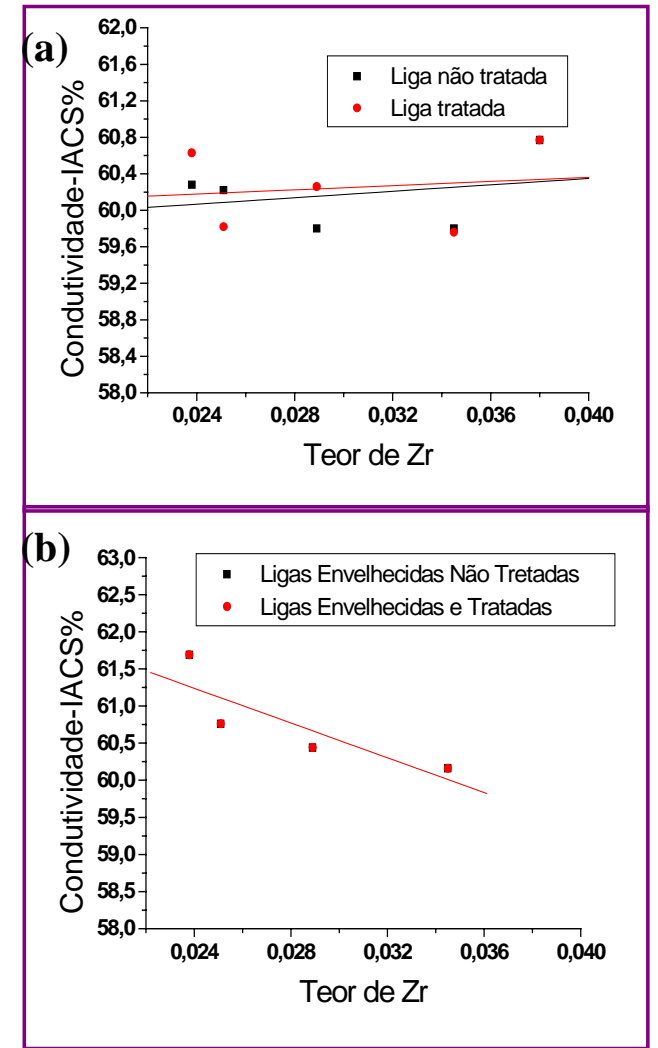

Figura 7 - Valores de condutividade elétrica com e sem teste de termorresistividade em função do teor de $\mathrm{Zr}$, antes e após envelhecimento de 1.5 ano, respectivamente.

Os valores encontrados no ensaio de condutividade elétrica, para as situações propostas, com e sem tratamento térmico, Figura 7A, confirmam o que se constatou na avaliação do aspecto da fratura. Não se constata modificação significativa na região da fratura, os dimples das microcavidades só apresentam pequenas diferenças quando se muda o teor de $\mathrm{Zr}$.

Os valores observados na Figura 7B, com e sem envelhecimento artificial, mostram que o efeito da temperatura e do tempo, sobre o mesmo teor de $\mathrm{Zr}$, leva ao entendimento de que o alívio de tensão promovido visa observar a mobilidade do $\mathrm{Zr}$ no alumínio. E, pelos valores encontrados para a condutividade elétrica pode se entender que esta mobilidade é baixa, não havendo modificação significativa nesta propriedade apesar de diminuir com o teor de Zr.

O teste de termorresistividade e o envelhecimento natural não acarretaram qualquer alteração nos valores de condutividade elétrica, evidenciando a total imparcialidade desta propriedade.

\section{CONCLUSÃO}

O efeito da temperatura e do tempo parece refletir sobre os valores dos LRT no sentido de acomodar a estrutura interna, aliviando as tensões oriundas das etapas da deformação a que foram submetidas às amostras. Mesmo assim, os gráficos das Figuras 5 e 6 indicam que o teor crescente de $\mathrm{Zr}$ tende a elevar o LRT destas ligas, para níveis aceitáveis para aplicação em cabos Tx ou Dx.

Os valores encontrados no ensaio de condutividade elétrica sugerem uma variação mínima de condutividade mediante o aumento do teor de $\mathrm{Zr}$ para a faixa estudada, além de manter-se com valores aceitáveis para aplicação elétrica. Assim, mais uma vez, tem-se um indício de que o sistema mesmo não apresentando mudança estrutural, caso do teste de termorresistividade ou apresentando mudanças estruturais caso do envelhecimento natural, suas características se mantém aceitáveis.

A semelhança das micrografias obtidas no MEV bem como as análises por EDS vêm a indicar boa neutralidade das ligas produzidas, mesmo após 1,5 ano de envelhecimento natural, sem modificações estruturais ou surgimento de precipitado expressivo.

As ligas produzidas mostraram bom desempenho nos diversos ensaios realizados, gerando perspectivas animadoras para a sua aplicação, já que os requisitos para a termorresistividade analisados foram atingidos.

\section{AGRADECIMENTOS}

Os Autores agradecem o apoio recebido para o sucesso desta tarefa a UFPA, ELETRONORTE e a ALUBAR METAIS S/A.

\section{REFERÊNCIAS BIBLIOGRÁFICAS}

(1) MIRANDA, G. C.; GIUDICE FILHO, E. B.; RODRIGUES, B. J. F.; 
NASCIMENTO, C. A. M.; BRAGA, G. E.; UEDA, S. M. - O Estado da Arte na Aplicação de Condutores Compactos Em Projetos De Linhas Aéreas De Transmissão - Aspectos Mecânicos e Elétricos para o Novo Modelo Desenvolvido no Brasil, SNPTEE Seminário Nacionalde Produção e Transmissão ee Energia Elétrica, Grupo de Estudo de Linhas de Transmissão GLT - 08, Curitiba - Paraná, 16 a 21 Outubro de 2005.

(2) DOMINGUES, I. T.; LOPES, J. C. R.; CABRAL S.; ROQUELANE R.; UEDA, S. M., ANAUATE S. Emprego de Novas Tecnologias de Materiais em Linhas de Transmissão Aérea com a Substituisão de Condutores ACSR por Condutores Termorresistentes TACSR e TACIR (Invariável)"-XVIII SNPTEE, Curitiba, PR, 16 a 21 de outubro de 2005.

(3) NASCIMENTO, C. A. M et al.; GIUDICE, E. B.; MOURÃO, M. A.; BRITO, J. M. C.; Assunção, J. M.;
FONSECA, B. Q. A.; FERREIRA, V. O. A. H. L.; BRACARENSE, A. Q.; UEDA S. M. -Aumento da Capacidade de Transmissão de Linhas Aéreas, Utilizando Cabo CAA de Alumínio Termorresistente (TAL) - XV SNPTEE, Foz do Iguaçu, PR, 17 a 22 de outubro de 1999.

(4) FURUKAWA, Informativo Técnico No. 3, Liga de Alumínio Termorresistente, outubro/1999.

(5) ROBSON, J. D. and PRANGNELL, P. B. - Mat. Sci. Tech., 18(6):607, 2002.

(6) MORERE , B.; C., MAURICE, SHAHANI, R.. and DRIVEr, J. - Metall. Mater. Trans. A, 32:625, 2001.

7) RANGANATHAN, K.; LAST, H. R., and SANDERS, T. H. - In Proc. 3rd Int. Conf. Aluminum Alloys (ICAA-3), volume 2, page 15, 1992.

(8) NBR 7272/1988. 